SANDIA REPORT

SAND2010-8119

Unlimited Release

Printed November 2010

\title{
Main Group Adducts of Carbon Dioxide and Related Chemistry (LDRD 149938)
}

\author{
Diane A. Dickie, Constantine A. Stewart, Brian M. Barry, and Richard A. Kemp
}

Prepared by

Sandia National Laboratories

Albuquerque, New Mexico 87185 and Livermore, California 94550

Sandia National Laboratories is a multi-program laboratory managed and operated by Sandia Corporation,

a wholly owned subsidiary of Lockheed Martin Corporation, for the U.S. Department of Energy's

National Nuclear Security Administration under contract DE-AC04-94AL85000.

Approved for public release; further dissemination unlimited. 
Issued by Sandia National Laboratories, operated for the United States Department of Energy by Sandia Corporation.

NOTICE: This report was prepared as an account of work sponsored by an agency of the United States Government. Neither the United States Government, nor any agency thereof, nor any of their employees, nor any of their contractors, subcontractors, or their employees, make any warranty, express or implied, or assume any legal liability or responsibility for the accuracy, completeness, or usefulness of any information, apparatus, product, or process disclosed, or represent that its use would not infringe privately owned rights. Reference herein to any specific commercial product, process, or service by trade name, trademark, manufacturer, or otherwise, does not necessarily constitute or imply its endorsement, recommendation, or favoring by the United States Government, any agency thereof, or any of their contractors or subcontractors. The views and opinions expressed herein do not necessarily state or reflect those of the United States Government, any agency thereof, or any of their contractors.

Printed in the United States of America. This report has been reproduced directly from the best available copy.

Available to DOE and DOE contractors from

U.S. Department of Energy

Office of Scientific and Technical Information

P.O. Box 62

Oak Ridge, TN 37831

Telephone: $\quad$ (865) 576-8401

Facsimile: (865) 576-5728

E-Mail:_reports@adonis.osti.gov

Online ordering: http://www.osti.gov/bridge

Available to the public from

U.S. Department of Commerce

National Technical Information Service

5285 Port Royal Rd.

Springfield, VA 22161

Telephone: $\quad$ (800) 553-6847

Facsimile: (703) 605-6900

E-Mail: $\quad$ orders@ntis.fedworld.gov

Online order: $\quad$ http://www.ntis.gov/help/ordermethods.asp?loc=7-4-0\#online

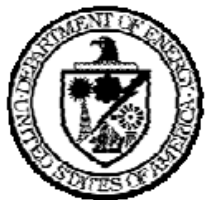


SAND2010-8119

Unlimited Release

Printed November 2010

\title{
Main Group Adducts of Carbon Dioxide and Related Ligand Chemistry (LDRD 149938)
}

\author{
Diane A. Dickie ${ }^{a}$, Constantine A. Stewart ${ }^{b}$, Brian M. Barry ${ }^{a}$, Richard A. Kemp ${ }^{\text {a,b }}$ \\ ${ }^{a}$ Department of Chemistry and Chemical Biology, University of New Mexico, MSC03 \\ 2060, Albuquerque NM 87131 \\ ${ }^{\mathrm{b}}$ Ceramics Processing and Inorganic Materials Department, Sandia National Laboratories, \\ P.O. 5800, Albuquerque NM 87185-1349
}

\begin{abstract}
This late-start LDRD was broadly focused on the synthetic attempts to prepare novel ligands as complexing agents for main group metals for the sequestration of $\mathrm{CO}_{2}$. In prior work we have shown that certain main group (p block elements) metals such as tin and zinc, when ligated to phosphinoamido- ligands, can bind $\mathrm{CO}_{2}$ in a novel fashion. Rather than simple insertion into the metal-nitrogen bonds to form carbamates, we have seen the highly unusual complexation of $\mathrm{CO}_{2}$ in a mode that is more similar to a chemical "adduct" rather than complexation schemes that have been observed previously. The overarching goal in this work is to prepare more of these complexes that can a) sequester (or bind) $\mathrm{CO}_{2}$ easily in this adduct form, and b) be stable to chemical or electrochemical reduction designed to convert the $\mathrm{CO}_{2}$ to useful fuels or fuel precursors. The currentlyused phosphinoamido- ligands appear at this point to be less-stable than desired under electrochemical reduction conditions. This instability is believed due to the more delicate, reactive nature of the ligand framework system. In order to successfully capture and convert $\mathrm{CO}_{2}$ to useful organics, this instability must be addressed and solved. Work described in the late-start LDRD was designed to screen a variety of ligand/metal complexes that a priori are believed to be more stable to polar solvents and possible mild hydrolytic conditions than are the phosphinoamido- ligands. Results from ligand syntheses and metal complexation studies are reported.
\end{abstract}




\section{Acknowledgements}

The authors would like to thank the National Science Foundation for funding initial work in this area. We would also very much like to thank Mr. John A. Emerson for highly useful and productive conversations prior to beginning this work. His help in getting this LDRD funded is greatly appreciated. 


\section{Table of Contents}

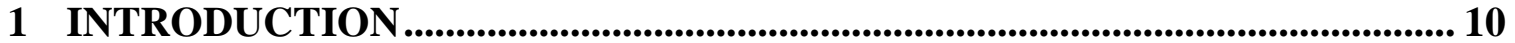

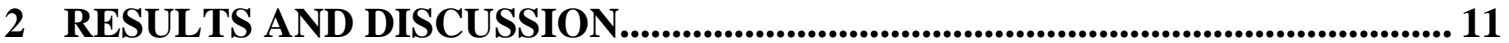

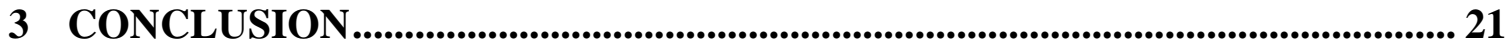

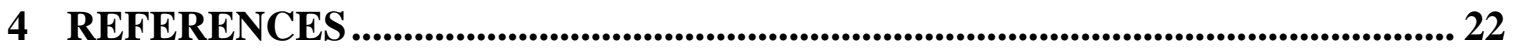

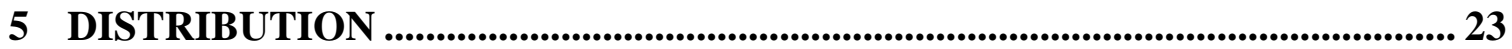




\section{LIST OF FIGURES}

Figure 2.1.1 Diagram illustrating the electron density locations and bond polarities found in $\mathrm{CO}_{2}$. The electrophilic/nucleophilic sites determine reactivity trends for $\mathrm{CO}_{2} \ldots \ldots \ldots . . .12 \#$ Figure 2.5.1 Possible amphoteric ligand structures based on a N-OH framework.......... 17\# Figure 2.5.2 Reaction of 8-hydroxyquinoline with $\mathrm{SnCl}_{2}$ to give $\mathrm{Sn}$ (quinolin-8-olato) ${ }_{2} 18 \#$ Figure 2.5.3 Crystal structure of Sn(quinolin-8-olato) 2 ................................................ 18\#

Figure 2.6.1 Possible ligand structures based on M-C bound frameworks....................... 19\#

Figure 2.6.2 Route to bis(pyridyl) metal species........................................................ 20\#

Figure 2.6.3 Route to bis(pyridyl) metal species. ........................................................... 21\# 


\section{Abbreviations}

$\begin{array}{ll}\mathrm{CO}_{2} & \text { Carbon dioxide } \\ \text { DOE } & \text { Department of Energy } \\ \text { HMPA } & \text { Hexamethylphosphoramide } \\ \text { IR } & \text { Infrared spectroscopy } \\ \text { LDRD } & \text { Laboratory Directed Research and Development } \\ \text { NMR } & \text { Nuclear magnetic resonance } \\ \text { SNL } & \text { Sandia National Laboratories } \\ \text { TMEDA } & \text { Tetramethylethylenediamine }\end{array}$




\section{Main Group Adducts of Carbon Dioxide and Related Ligand Chemistry (LDRD 149938)}

The goal of this 6-month, late-start LDRD project was to investigate the design, synthesis and characterization, and metal complexation of a number of new ligands that might prove useful for the possible sequestration and conversion of $\mathrm{CO}_{2}$. Recently, we have shown that $\mathrm{CO}_{2}$ at atmospheric pressure under simple bubbling can form "adducts" with certain main group metal - ligand compounds. While almost all ongoing research directed towards the fixation of $\mathrm{CO}_{2}$ involves transition metals, we have chosen to examine main group metals. Main group metals are also called p-block elements, indicating that the valence electrons used in binding ligands are in $p$ orbitals, not the $d$ orbitals used in transition metals. While transition metals range in cost from relatively inexpensive (Fe, $\mathrm{Co}, \mathrm{Ni}$, etc.) to extremely expensive and rare ( $\mathrm{Rh}, \mathrm{Ir}, \mathrm{Pd}, \mathrm{Pt}$, etc.), the main group metals such as tin and zinc we are interested in exploiting are quite inexpensive and plentiful. In general, when main group metals are complexed with amido-type (nitrogen-bound) ligands, $\mathrm{CO}_{2}$ is known to insert into the $\mathrm{M}-\mathrm{N}$ bond to form organic carbamates. While this reaction is of interest to produce carbamates, $\mathrm{CO}_{2}$ plays only a role of inserting species - the types of organic product derived from $\mathrm{CO}_{2}$ are quite limited in scope. As well, simple insertion of $\mathrm{CO}_{2}$ into activated $\mathrm{M}-\mathrm{N}$ bonds, or other $\mathrm{M}$ element bonds, cannot be a catalytic process of $\mathrm{CO}_{2}$ conversion. Our goal in this research is to examine these new "adducts" of $\mathrm{CO}_{2}$ with main group complexes in order to convert $\mathrm{CO}_{2}$ via chemical or electrochemical means to organic fuels or fuel precursors, preferably catalytically. In our initial samples of complexed $\mathrm{CO}_{2}$ with main group complexes, ones in which the concept of $\mathrm{CO}_{2}$ adduct formation was first demonstrated, the phosphinoamido- ligands appear to be less stable than required to allow reduction of $\mathrm{CO}_{2}$. The entire issue of ligand and adduct stability is a delicate one. For example, one wants the $\mathrm{CO}_{2}$ adduct of the metal complex to be stable, yet it cannot be so stable that it is unreactive towards reductants. So, there is a delicate balance between stability and instability that drives every complex that is formed, and every possible catalytic reaction that is done. If complexes are too stable, they can become thermodynamic "dead ends" in a catalytic conversion cycle, while if the complexes are too unstable, they do not exist long enough to perform useful reductive chemistry upon them. As well, the structural integrity of the ligand framework must also stand up under the reaction conditions used. Ligands that fall apart easily are of little use other than as sources to possibly demonstrate fundamental concepts for the complex. Conversion under electrochemical conditions requires that the metal complex, including the ligand framework, must be stable under electrochemical conditions. Unfortunately, our initial complexes that demonstrated our adduct concept are not stable enough to undergo electrochemical reduction. The work presented in this SAND report documents our initial thoughts and efforts towards the syntheses of new and novel ligands with improved stability. Also included are the synthetic strategies employed to manufacture these metal complexes using these ligands, as well as initial $\mathrm{CO}_{2}$ complexation studies. Essentially all of our ligands and metal complexes are well-characterized, often by single crystal X-ray diffraction in addition to the typical analytical techniques used such as NMR and IR spectroscopies. 
Significant research goals and milestones accomplished over the course of this late-start LDRD include:

0 Synthesis and characterization of $\mathrm{Sn}$ and $\mathrm{Zn}$-based $\mathrm{CO}_{2}$ adducts. The inspirational chemical targets of this LDRD work were prepared and well-characterized. Although some of the work to prepare these compounds was performed earlier, further information on these new compounds is presented.

o Initial attempts at electrochemical reduction of a Sn-based $\mathrm{CO}_{2}$ adduct. The initial attempts at electrochemical reduction of a Sn-CO2 adduct were performed. The conclusions from these preliminary experiments indicate that the ligand backbone must be stabilized in order to obtain reproducible and clean results.

o New ligand frameworks were designed. Based on the preliminary electrochemical experiments it was determined that new ligand frameworks were required. Design and synthesis of several new framework structures were initiated and performed during this time.

o New metal complexes were prepared and characterized during this period. New ligands were complexed to various main group metals to form new complexes. These metal-complexes were also characterized by traditional methods, including single crystal X-ray crystallography. 


\section{Introduction}

Carbon dioxide $\left(\mathrm{CO}_{2}\right)$ is a relatively "useless" molecule other than as a carbon source, and so the conversion of $\mathrm{CO}_{2}$ into useful chemicals and fuels is a topic of worldwide interest as the need to mitigate global warming becomes more urgent. ${ }^{1-5}$ In a current project, Sandia has a major research thrust in reducing $\mathrm{CO}_{2}$ levels by using hightemperature, solar methods. ${ }^{6,7}$ Reduction of $\mathrm{CO}_{2}$ at lower temperatures, ideally ambient temperature, would be a major scientific breakthrough and assembling a catalytic process for this transformation would be a technological game-changer for $\mathrm{CO}_{2}$ fixation. Therefore, emphasis on a single, high-temperature approach from Sandia is not adequate to ensure success in this arena of $\mathrm{CO}_{2}$ activation. Additionally, Sandia has LDRD programs examining the capture and chemical sequestration of $\mathrm{CO}_{2}$ via a number of routes, primarily using heterogeneous or polymeric supports. In the work reported here our goal is to combine aspects of these two Sandia interests by developing a novel approach to capturing and reducing $\mathrm{CO}_{2}$. The overall concept is based on a generalized type of metal- $\mathrm{CO}_{2}$ "adduct" that we have discovered and characterized very recently at Sandia/UNM. It is known that direct electrochemical reduction of $\mathrm{CO}_{2}$ is disfavored largely due to the energy required to "bend" the linear $\mathrm{CO}_{2}$ molecule after addition of electrons. Our proposed approach is geared towards overcoming the primary barrier of efficiently reducing $\mathrm{CO}_{2}$ electrochemically - that of the energy requirement to "bend" $\mathrm{CO}_{2}$ after addition of these electrons - by forming coordination adducts of $\mathrm{CO}_{2}$ that "prebend" $\mathrm{CO}_{2}$ using electroactive metal catalysts. Moreover, the metals used in our suggested experiments are inexpensive and readily-available, and the $\mathrm{CO}_{2}$-adduct formation reaction takes place at room temperature.

This report describes the efforts from the end of March 2010 to the end of September 2010 that were funded by a late-start Laboratory Directed Research and Development (LDRD 149938) project to investigate new ligands that might be useful for preparing metal- $\mathrm{CO}_{2}$ adducts. As well, metal complexes of these new ligands were prepared and characterized. Initial electrochemical reduction studies of metal- $\mathrm{CO}_{2}$ adducts were also performed. 


\section{Results and Discussion}

\subsection{Fundamental Description of $\mathrm{CO}_{2}$}

Carbon dioxide $\left(\mathrm{CO}_{2}\right)$, the fully oxidized form of carbon, is an energetically "spent" molecule, although this does not imply that it is non-reactive. Far from it, as $\mathrm{CO}_{2}$ is known to undergo a wide range of reactions with various other reagents. ${ }^{1}$ However, generally speaking the other reagent used must be activated in some way. $\mathrm{CO}_{2}$ is an amphoteric molecule, capable of acting as a Lewis acid by attack at the electrophilic central carbon atom, or acting as a Lewis base by donation from the lone pairs of electrons located on the terminal $\mathrm{O}$ atoms. The diagram shown in Figure 2.1.1 illustrates this bond polarity difference, and clearly shows the modes of expected reactivities at the carbon or oxygen sites.

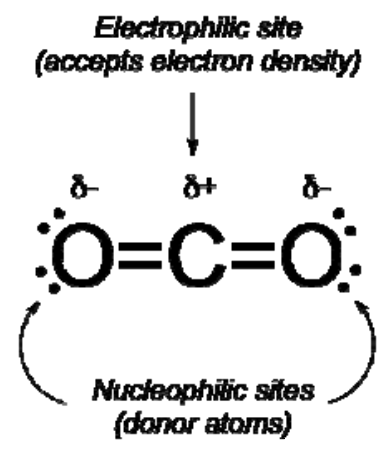

Figure 2.1.1 Diagram illustrating the electron density locations and bond polarities found in $\mathrm{CO}_{2}$. The electrophilic/nucleophilic sites determine reactivity trends for $\mathrm{CO}_{2}$.

A classic, long-known example demonstrating the electrophilic nature of $\mathrm{CO}_{2}$ is the quenching of Grignard reagents (organomagnesium species) with dry ice (solid $\mathrm{CO}_{2}$ ) to produce organic acids after workup. ${ }^{8}$ The nucleophilic organic anions attack the central carbon atom to form carboxylates, which can be converted to organic acids upon protonation. As an example of further reactivity germane to this report, $\mathrm{CO}_{2}$ is known to insert into various polarized metal-element bonds such as metal-alkoxides or metalamides to form important organic precursors such as metal carbonates ( $\left.\mathbf{M}-\mathbf{C O}_{2}-\mathrm{OR}\right)$ or carbamates $\left(\mathrm{M}-\mathbf{C O}_{2}-\mathrm{NR}_{2}\right)$. ${ }^{9}$ The insertion mode of reactivity for $\mathrm{CO}_{2}$ to form highly thermodynamically stable products is commonly observed, and is unfortunately an undesired alternative reaction pathway in the research described in this report.

\subsection{Main Group Metal- $\mathrm{CO}_{2}$ Adducts}

Recently, we have discovered a new bonding mode for $\mathrm{CO}_{2}$ towards main group metals that is to our knowledge unprecedented in the literature. Main group metals are those metals located on the right-hand side of the periodic table that utilize $s$ and $p$ orbitals as 
the outer bonding valence shell. These metals - examples including tin, zinc, bismuth, lead, silicon, and so forth - differ significantly in reactivity from the transition metals, which use $d$ orbitals for binding to ligands. In two species based on $\mathrm{Sn}$ and $\mathrm{Zn}$ and using modified amido ligands, we have shown the extraordinary binding of $\mathrm{CO}_{2}$ as a Lewis acid-base adduct to the metal complexes. These two species, briefly discussed in the next paragraph, form the origin for the work performed in this late-start LDRD.

We have previously been interested in the insertion reactions of $\mathrm{CO}_{2}$ into various maingroup metal amides to form metal-containing carbamates. ${ }^{10-12}$ In these studies, the insertion of $\mathrm{CO}_{2}$ was desired in order to form unstable species that could upon warming eliminate isocyanates and/or carbodiimides as organic reaction products. This reaction scheme, based on work by Sita, ${ }^{13}$ to form these species is shown in Figure 2.2.1 below.

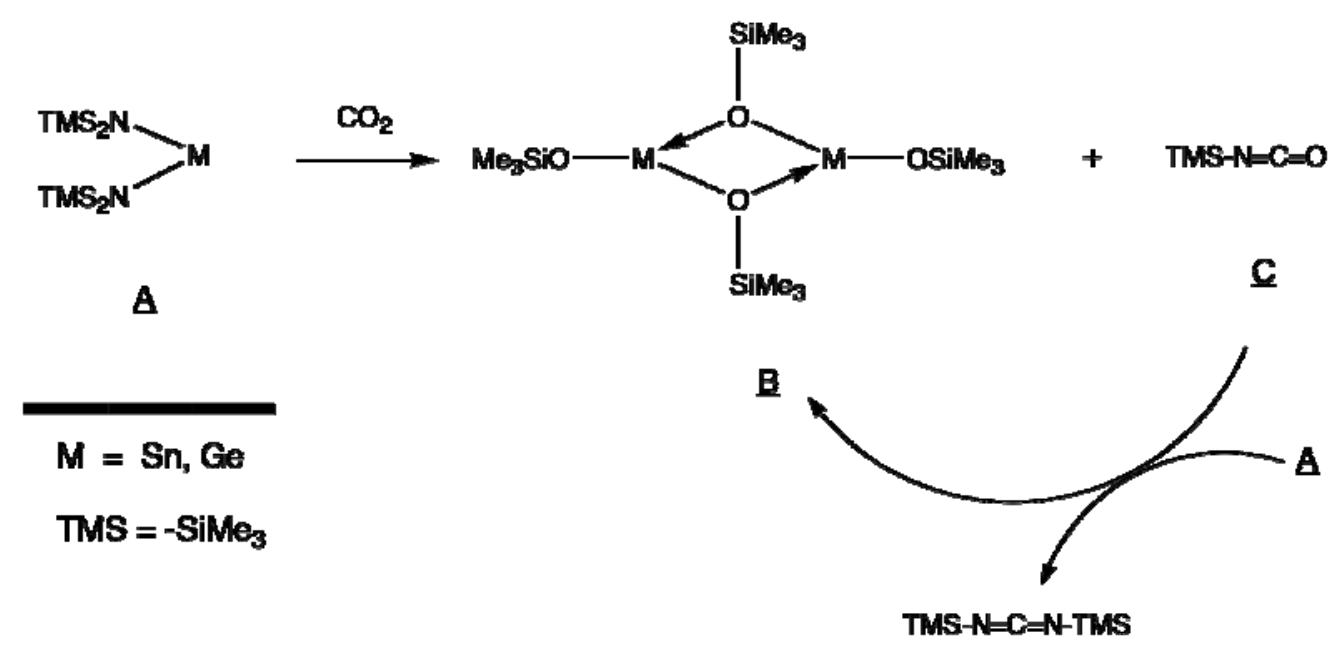

Figure 2.2.1 Reaction scheme demonstrating the insertion of $\mathrm{CO}_{2}$ into main group metal amides to form isocyanates and/or carbodiimides.

A key element of the above scheme is the direct insertion of $\mathrm{CO}_{2}$ into the $\mathrm{M}-\mathrm{N}$ bond to form a metal carbamate, which then eliminates an $\mathrm{R}-\mathrm{N}=\mathrm{C}=\mathrm{O}$ species (isocyanate) while concomitantly forming a strong $\mathrm{M}-\mathrm{OSiMe}_{3}$ bond to help drive the reaction forward. A secondary reaction in which the isocyanate inserts in place of $\mathrm{CO}_{2}$ leads to the carbodiimide. Our academic group at the University of New Mexico has published widely on this topic. However, in the screening of various modified-amido ligands we unexpectedly discovered the formation of a new bonding mode for $\mathrm{CO}_{2}$ - that of a main group metal- $-\mathrm{CO}_{2}$ adduct, rather than the expected inserted/eliminated product.

We utilized the $\left[\left(\mathrm{R}_{2} \mathrm{P}\right)\left(\mathrm{Me}_{3} \mathrm{Si}\right) \mathrm{NH}\right]$ phosphino-modified amido ligand, and the dinuclear(ligand)zinc complex was prepared using an alkane elimination reaction with $\mathrm{ZnEt}_{2}$ that did not go to completion. However, addition of $\mathrm{CO}_{2}$ led to the final product as shown. A classic metathesis route was used to prepare a related divalent Sn complex 
that used a similar phosphino-modified amido ligand, and in this case the bis(ligand) Sn species could be formed. Rather than inserting into the metal-nitrogen bond as was expected, we instead observed that $\mathrm{CO}_{2}$ formed a side-on bound "adduct" with the metalcontaining complex after simple bubbling of $\mathrm{CO}_{2}$ through either metal complex solution at room temperature. The example of this reaction type using zinc is shown in equation form in Figure 2.2.2, and we have confidently identified the product by single crystal Xray diffraction, as shown below in Figure 2.2.3. We also showed a very similar structure for $\mathrm{Sn}$ as well, although it is not shown. What makes this reaction extremely interesting is that the expected $\mathrm{CO}_{2}$ insertion into both $\mathrm{Zn}-\mathrm{N}$ bonds to form carbamates is not observed, but rather a bent $\mathrm{CO}_{2}$ adduct is formed, using two Lewis base/acid pairs for

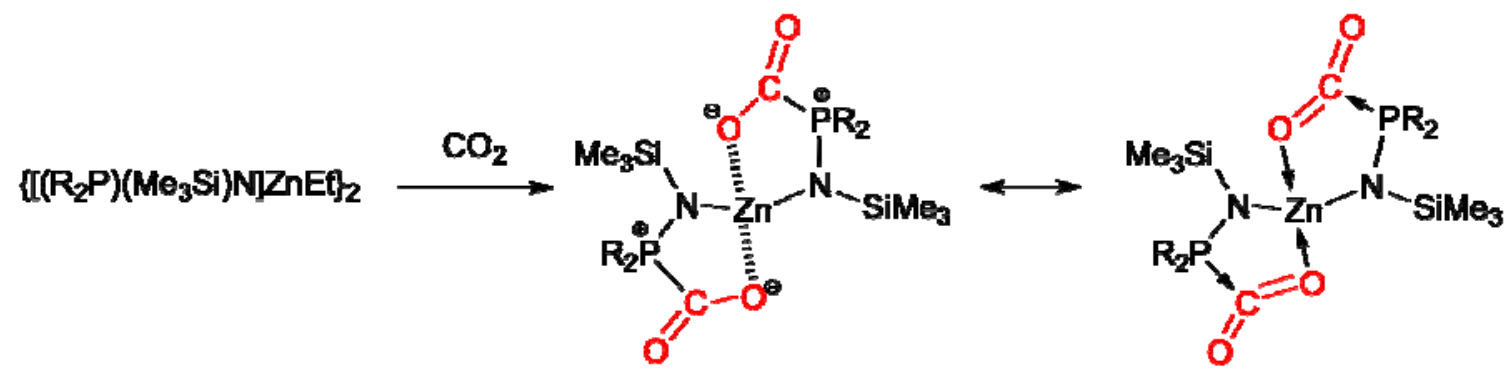

A

B

Figure 2.2.2 Synthetic scheme used to prepare $\mathrm{Zn}-\mathrm{CO}_{2}$ adduct.

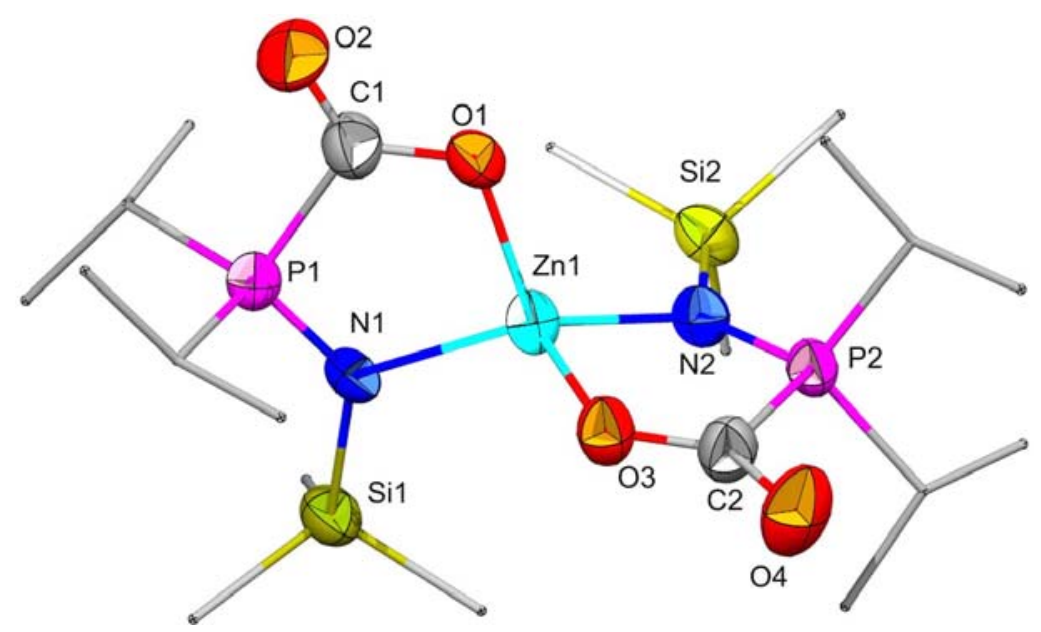

Figure 2.2.3 Thermal ellipsoid plot of $\mathrm{Zn}-\mathrm{CO}_{2}$ adduct. 
complexation. This bonding mode can be seen more clearly in resonance structure B above with use of the four arrows to denote Lewis acid/base pairs, although we note that zwitterionic resonance form A can also describe the molecule. As mentioned, we have also observed this same reactivity mode using $\mathrm{Sn}^{2+}$ and related ligands to produce an acid/base adduct. We are interested in further expanding the number and types of complexes shown by varying the metals and ligand structures by examining inexpensive and readily-available metals such as tin, zinc, and others in Groups 12-15, and to design more robust ligands. Almost all research efforts involving $\mathrm{CO}_{2}$ activation utilize transition metals, and so one of the unique aspects of this approach is the focus will be put on these main group elements. It is apparent from the crystal structures that the $\mathrm{CO}_{2}$ adducts are significantly bent with $\mathrm{O}-\mathrm{C}-\mathrm{O}$ angles of $\sim 130^{\circ}$. We hypothesize that these adduct complexes of $\mathrm{CO}_{2}$ will electrochemically accept electrons kinetically more easily than will linear $\mathrm{CO}_{2}$ since the complexes are already bent upon complexation to the metal. This should allow more facile entry of the electrons into the $\mathrm{CO}_{2}$ fragment to form radical anions.

\subsection{Initial Electrochemical Investigation of Main Group Metal-CO $\mathrm{CO}_{2}$ Adduct}

The $\mathrm{Sn}-\mathrm{CO}_{2}$ adduct described above is relatively stable, although sitting at room temperature over a 2-3 day period will lead to elimination of organic isocyanates and other unidentified products. Thus, there is a delicate balance in the stability of this adduct. The Zn-adduct is similar in behavior, although it is more stable. This gentle instability is encouraging, as it is not desired to have the complex adducts be too stable. In our model, the adduct must exist long enough to do the reductive chemistry but not be so stable that the energy required to reduce the $\mathrm{CO}_{2}$ is too great. Samples of the $\mathrm{Zn}-\mathrm{CO}_{2}$ adduct were sent to Professor William Geiger of the University of Vermont for electrochemical studies. In a brief series of initial electrochemical experiments into the $\mathrm{Zn}-\mathrm{CO}_{2}$ adduct it was discovered that the ligand framework did not appear to be chemically robust enough to survive the supporting electrolyte solution $\left(\left[\mathrm{Bu}_{4} \mathrm{~N}\right]\left[\mathrm{PF}_{6}\right]\right.$ in tetrahydrofuran) and electrochemical experiments.

The targeted reduction occurs just before the solvent limit and creates problems to the electrode surface. However, the compound is definitely reducible. The reduction can only be seen by using a gold electrode, with either Pt or glassy carbon showing no wave at all. After one scan, the gold electrode is at least partially passivated, so that nothing is seen on a second scan. If the electrode is taken outside the box and polished, the wave reappears in a new scan of the solution. This is typical of a system in which the reduction product coats the electrode and will pose a problem if we want to do bulk electrolysis. The potential of the reduction (irreversible) is $-3.25 \mathrm{v} v \mathrm{v}$. FcH. A typical reduction scan of the $\mathrm{Zn}-\mathrm{CO}_{2}$ adduct is shown in Figure 2.3.1. Initially, it appears that the ligand is degrading during reaction. 


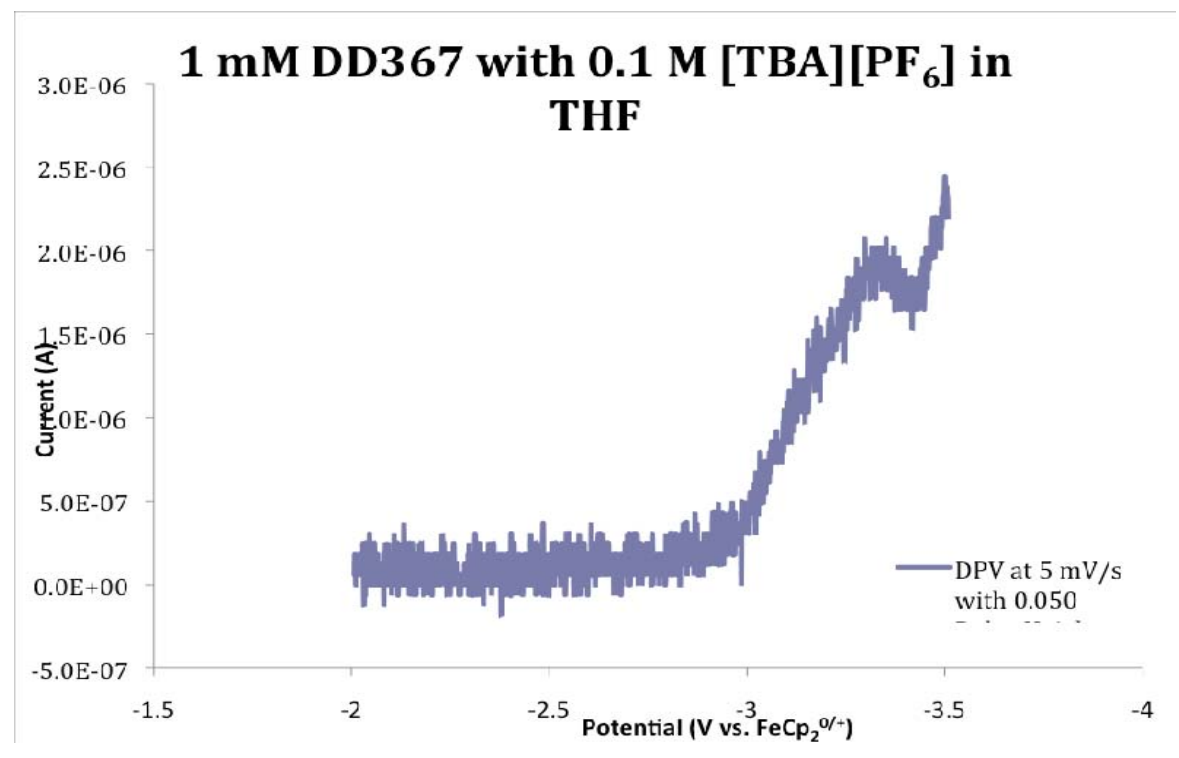

Figure 2.3.1 Electrochemical reduction scan of $\mathrm{Zn}-\mathrm{CO}_{2}$ adduct in $[\mathrm{TBA}]\left[\mathrm{PF}_{6}\right] / \mathrm{THF}$ solution.

While this is disappointing, it is not totally surprising as the ligand framework consists of relatively delicate and reactive $\mathrm{Zn}-\mathrm{N}, \mathrm{N}-\mathrm{Si}$, and N-P bonds. These highly-polarized bonds are susceptible to a variety of reactions, including reaction with any miniscule sources of protons or base. The initial results from the electrochemistry experiments indicated to us that the stability of the ligand must be improved, and the rest of this report will document our attempts at preparing new, more chemically-robust ligands that can function in the same manner as the more delicate -[N($\left.\left(\mathrm{SiMe}_{3}\right)\left(\mathrm{PR}_{2}\right)\right]$ ligands.

\subsection{Introduction to the Design and Preparation of New Ligands}

When designing new ligands to prepare $\mathrm{CO}_{2}$ adducts from main group metal complexes, there are several features that can be generalized and will be common to all ligand types. First, the goal of the research is to prepare more stable ligands, so the use of reactive, highly-polarized bonds should be minimized. At first glance, one would wonder why reactive bonds in the frameworks of ligands should not be avoided totally, not just minimized. However, as an overall goal in this project we are also interested in understanding why these ligands behave as they do in forming metal- $\mathrm{CO}_{2}$ adducts in place of the expected inserted products, and as such we must also study the complexes (and related ligand structures) that we know will produce adducts. However, we have focused largely on new structures in ligands. Secondly, the new ligands must provide a site of Lewis basicity (likely a lone pair of electrons) that can attack the electrophilic carbon center in $\mathrm{CO}_{2}$ to form a bond or interaction. This feature is considered critical for the preparation of adducts. Thirdly, the location of the Lewis base site on the ligand must be close enough to the metal center to allow the nucleophilic $\mathrm{O}$ ends of the $\mathrm{CO}_{2}$ to coordinate to the Lewis acidic metal center. Design of various ligand frameworks will 
allow us to determine what is the optimal ring size for a coordinated $\mathrm{CO}_{2}$ adduct. The ligand must be capable of forming a strong interaction with the metal, and a strong metalligand bond must be present. As well, the ligand must be flexible enough to allow the coordinated $\mathrm{CO}_{2}$ to bend upon coordination, which is a key feature of these adducts. The rest of this report will summarize the ideas behind and synthetic chemistry of these new ligands.

\subsection{Mixed $\mathrm{N}-\mathrm{OH}$ Species as New Ligands}

In order to improve the stability of the ligands mixed $\mathrm{N}-\mathrm{OH}$ ligands have been prepared and treated with Sn(II) reagents. Several of these ligands are shown in Figure 2.5.1. All are compounds that can be expected (after conversion to the alkoxides) to bind to the metal through a more robust and stable $\mathrm{M}-\mathrm{O}$ bond. The Lewis base functionality of each new ligand is provided by an amine group that can provide the lone pair needed for binding to the electrophilic $\mathrm{C}$ atom of $\mathrm{CO}_{2}$.<smiles>Oc1cccc2cccnc12</smiles>

8-hydroxyquiniline

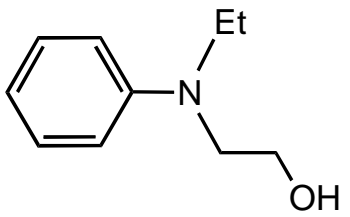

2-(N-ethylanilino) ethanol

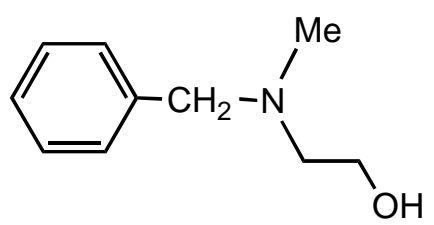

$N$-benzyl- $N$-methyl ethanolamine<smiles>O=C(O)c1ccc2ccccc2n1</smiles>

quinaldic acid<smiles>OCn1nnc2ccccc21</smiles>

1H-benzotriazole-1-methanol

Figure 2.5.1 Possible amphoteric ligand structures based on a N-OH framework.

The first Sn(II) complex prepared with these rings systems was bis(quinolin-8olato)tin(II) and was based on the method used by Kitamura and coworkers. ${ }^{14}$ The preparation of these types of complexes are in methanol. The complex was isolated in essentially quantitative yield (98\%) and the structure verified by ${ }^{1} \mathrm{H}$ and ${ }^{13} \mathrm{C}\left\{{ }^{1} \mathrm{H}\right\} \mathrm{NMR}$ spectroscopy and X-ray crystallography. The reaction scheme is shown in Figure 2.5.2 and the single crystal X-ray structure of the Sn complex is shown in Figure 2.5.3. 


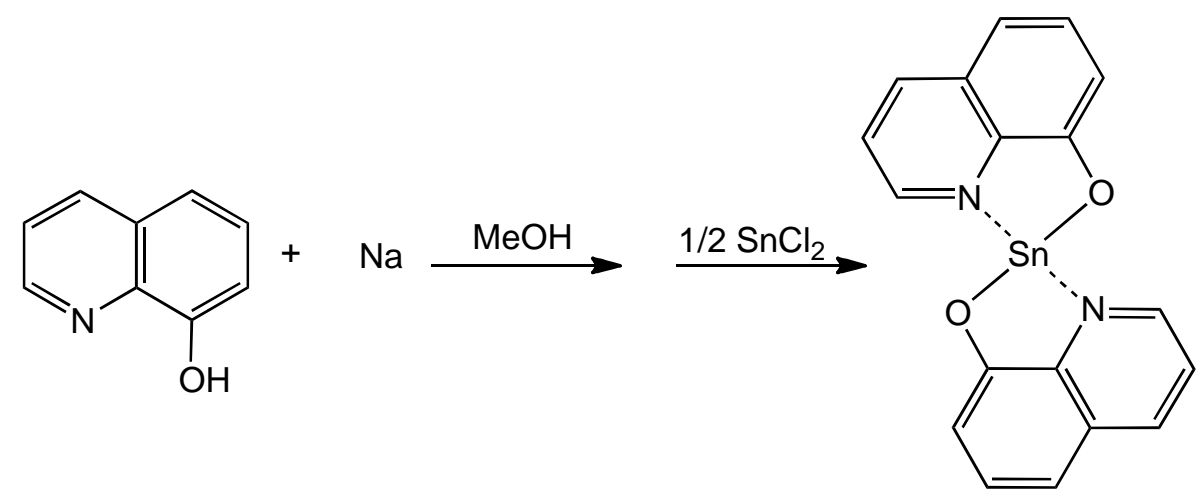

Figure 2.5.2 Reaction of 8-hydroxyquinoline with $\mathrm{SnCl}_{2}$ to give $\mathrm{Sn}$ (quinolin-8-olato) 2 .

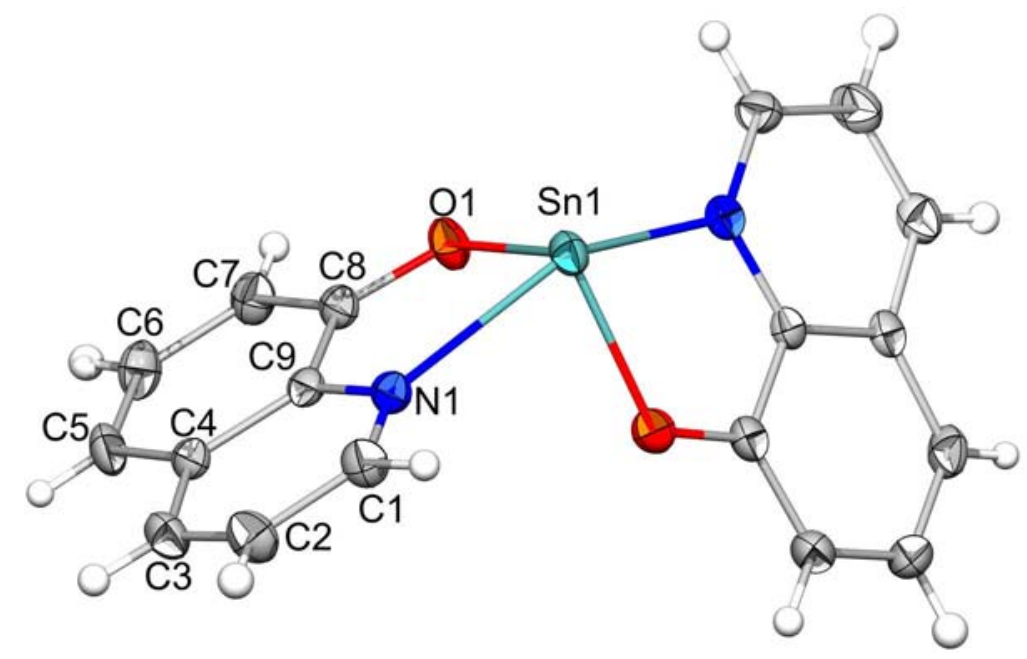

Figure 2.5.3 Crystal structure of Sn(quinolin-8-olato) ${ }_{2}$.

Bis(quinolin-8-olato)tin(II) was unfortunately not reactive towards $\mathrm{CO}_{2}$ in methanol at atmospheric pressure. However, when the pressure was raised to $90 \mathrm{psig}$ of $\mathrm{CO}_{2}$ the clear yellow solution in methanol of the complex turned cloudy after about 10 minutes. The IR of the isolated crude project indicated a new complex. Efforts to purify the product are currently underway.

The bis(benzotriazole-methoxy)tin(II) complex was prepared similarly to bis(quinolin-8olato)tin(II). Isolation of the crude project gave a light yellow solid from which clear colorless, very thin crystals were grown. Unfortunately they were too thin for X-ray crystallography determination. However, the ${ }^{1} \mathrm{H}$ NMR spectrum indicated that a $\mathrm{CO}_{2}$ complex had been prepared. Efforts are still in progress at the time of this writing to grow crystals suitable for X-ray diffraction studies and reactions with $\mathrm{CO}_{2}$ at atmosphere and at elevated pressures are also in progress. 
The attempted syntheses of bis( $N$-benzoyl- $N$-methyl-ethoxy-aniline)tin(II), bis(2-( $N$ ethylanilino)ethoxy)tin(II) and bis(2-(N-ethylanilino)ethoxy)tin(II) gave oils and oligomers. Considerable efforts were made to isolate and identify these products. The oils seem to show more promise and may eventually lead to isolation and identification.

The final complex in this ring series was with sodium salt of quindalic acid in methanol with $\mathrm{SnCl}_{2}$ to give the expected bis(2-(quinolino-carboxylate)tin(II). This reaction proceeded smoothly to give a crude yellow solid in good yield. Purification of this solid and identification of the complex is also underway at the time of this writing.

\subsection{Carbon-Metal Bound Species as New Ligands}

Another route to new ligands to be evaluated for the eventual formation of $\mathrm{CO}_{2}$-adducts with main group metals involves formation of direct metal-carbon bonds as the point of attachment. While M-C bonds do suffer some reactivity towards protic species, they can be more stable than their $\mathrm{M}-\mathrm{N}$ counterparts, particularly when using the main group metals. These new ligands that we have been working on still contain the "Lewis acidLewis base" pair functionalities that are required for preparing adducts. Examples of these types of structures are shown in Figure 2.6.1.
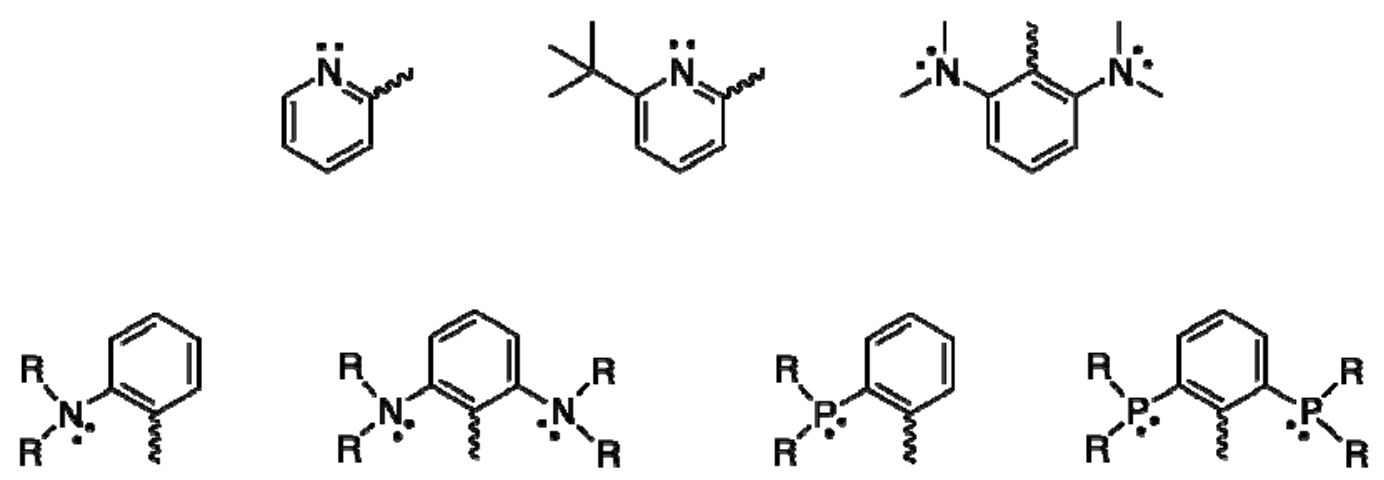

arv Indicates point of attachment to metal

Figure 2.6.1 Possible ligand structures based on M-C bound frameworks.

The ligands are all related by two structural features - the binding to metals by a metalcarbon bond, and the location of a lone pair of electrons on nitrogen or phosphorus near the binding site to the metal. The preparation of the ligands range in synthetic complexity from simple, one-step routes to difficult, multi-step procedures. Reactions that have been performed at the time of this writing will be discussed further. Preparation of other ligands will await further funding. 
The 2-pyridyl anion can be prepared by lithiating 2-bromopyridine at low temperature. This anion can then be added to $\mathrm{MX}_{2}(\mathrm{M}=\mathrm{Mg}, \mathrm{Sn}, \mathrm{Zn})$ to form a bis(pyridyl) metal complex after workup. The reaction scheme is shown in Figure 2.6.2.

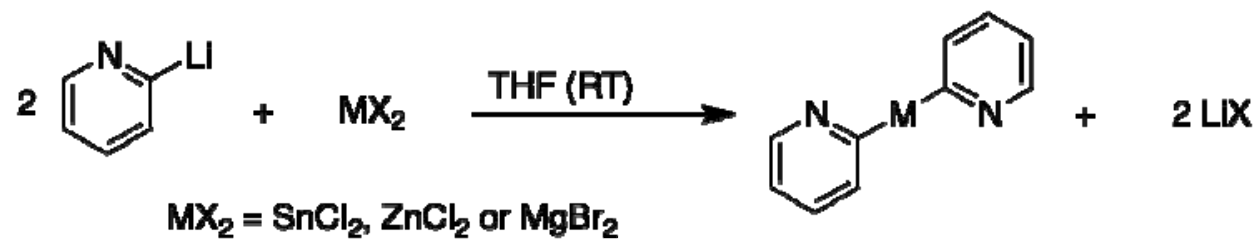

Figure 2.6.2 Route to bis(pyridyl) metal species.

While all of the species could be prepared, all were hampered by a severe lack of solubility in common organic solvents. The insolubility is most likely due to polymerization via the pyridyl nitrogen and a neighboring metal center. This has obviously prevented any NMR studies and further reaction with $\mathrm{CO}_{2}$ has not been investigated. Attempts to improve product solubility included heating the insoluble products in the presence of TMEDA or HMPA. Introduction of TMEDA or HMPA prior to salt elimination were attempted as well. These variations were unsuccessful at fixing solubility issues. Residues from filtrates (w/ or w/o TMEDA or HMPA) were recovered; however their NMR spectra were overly busy, and the weights of the residue were miniscule. In order to prepare a more-soluble version of these pyridyl ligands, we next attached a bulky $t$-butyl group adjacent to the $\mathrm{N}$ atom of the pyridine.

The $t$-butyl version of the pyridyl ligand was prepared in several steps starting with 2,6dibromopyridine. The multi-step reaction sequence for the preparation of t-butyl version is shown in Figure 2.6.3. The work up to obtain clean (by NMR) 6-t-butyl-2bromopyridine took 2.5 days and required $\sim 800 \mathrm{~mL}$ conc. $\mathrm{NH}_{4} \mathrm{OH}_{(\mathrm{aq})}$ and $\sim 800 \mathrm{~mL}$ of brine. This resulted in $\sim 2 \mathrm{~g}$ of product after distillation of the pure product. This process can only be scaled up by using even more $\mathrm{NH}_{4} \mathrm{OH}$ and brine and a larger separatory funnel (already using $1000 \mathrm{~mL}$ ).

All reactions resulted in translucent solutions which were pumped down resulting in oily solids. LiX salts were separated by dissolving the targeted products in toluene and filtering. LiX masses were reasonably close to expected weights (70-105\%). In contrast to the 2-pyridyl derivative, the addition of the $t$-butyl group did improve the solubility of the products significantly.

The washed Mg and Sn products resulted in oily solids whereas the Zn product is an orange powder. All products show t-butyl proton peaks with shifts differing from the position seen in 6-t-butyl-2-bromopyridine. Large, shallow, broad humps were often seen in the NMR spectra surrounding the $t$-butyl peak, and the pyryidyl region is full of an assortment of peaks. The Zn compound has two t-butyl peaks of approximately equal 
integration, which may suggest dimerization through a M-N interaction. The NMR of this proposed dimer also reveals a pyridyl:t-butyl integration of 1:2.5 (1:3 is ideal).

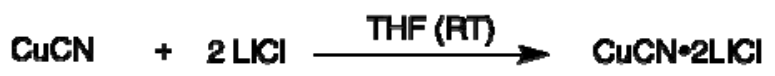

CuCN.2LICl + tBuL $\stackrel{\text { THF }\left(-85^{\circ} \mathrm{C}\right)}{\longrightarrow}$ tBuCuCN=UCI
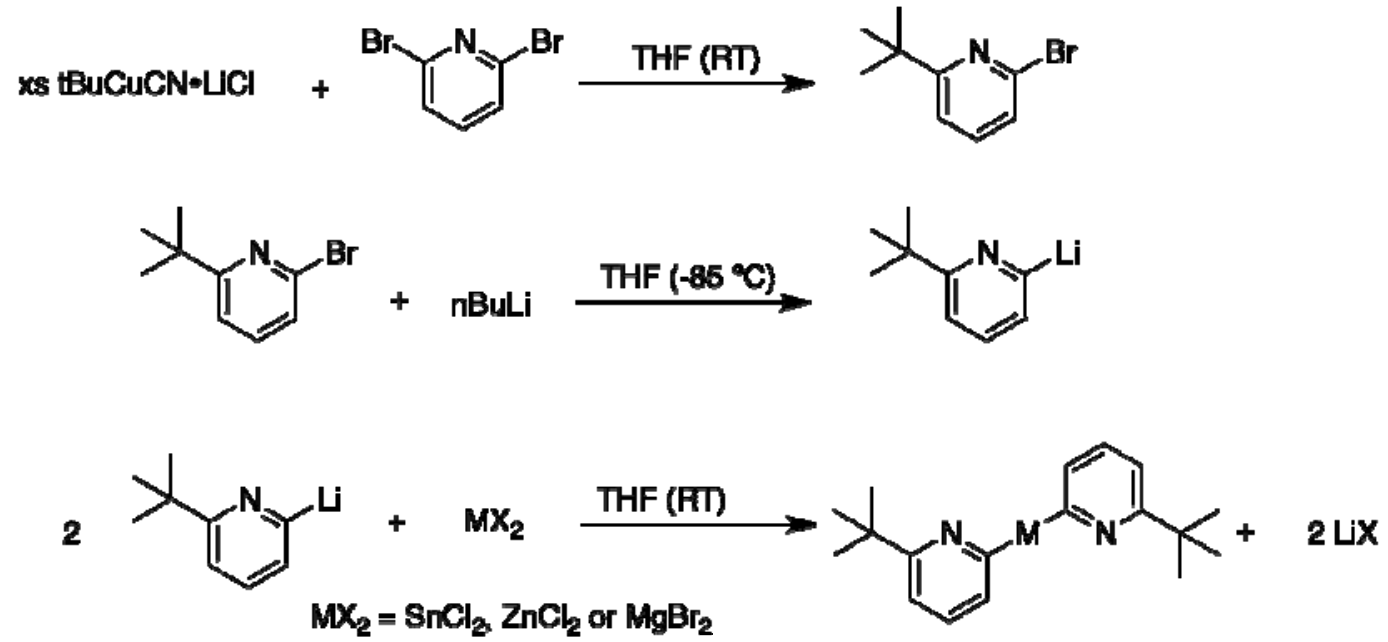

Figure 2.6.3 Route to bis(pyridyl) metal species.

These reactions have shown some promise both in improvements in solubility as shown by NMR; however, the NMR spectra are not clean and indicate a number of products are formed. This has led to problems in recrystallization, which has hampered the ability to obtain pure products. This is a major issue currently with the $t$-butyl derivatives.

Syntheses of the 2,6-dialkylaminopyridine ligands also require multiple steps. As of the writing of this report, we have prepared the 2,6-dimethylaminopyridine ligand by two different routes. While we first used the standard literature preparation, an improved route was developed by us that transferred the methyl groups to 2,6-diaminopyridine using $(\mathrm{MeO})_{3} \mathrm{P}=\mathrm{O}$ rather than excess $\mathrm{MeI}$ that was used previously. At the current time we have prepared the lithium salt of this ligand and reacted it with $\mathrm{SnCl}_{2}$ in an attempt to prepare the bis(ligand)Sn complex. 


\section{Conclusion}

This late-start LDRD was broadly focused on the synthetic attempts to prepare novel ligands as complexing agents for main group metals for the sequestration of $\mathrm{CO}_{2}$. In prior work we have shown that certain main group (p block elements) metals such as tin and zinc, when ligated to phosphinoamido- ligands, can bind $\mathrm{CO}_{2}$ in a novel fashion. Rather than simple insertion into the metal-nitrogen bonds to form carbamates, we have seen the highly unusual complexation of $\mathrm{CO}_{2}$ in a mode that is more similar to a chemical "adduct" rather than complexation schemes that have been observed previously. The overarching goal in this work is to prepare more of these complexes that can a) sequester (or bind) $\mathrm{CO}_{2}$ easily in this adduct form, and b) be stable to chemical or electrochemical reduction designed to convert the $\mathrm{CO}_{2}$ to useful fuels or fuel precursors. The currentlyused phosphinoamido- ligands appear at this point to be less-stable than desired under electrochemical reduction conditions. This instability is believed due to the more delicate, reactive nature of the ligand framework system. In order to successfully capture and convert $\mathrm{CO}_{2}$ to useful organics, this instability must be addressed and solved. Work described in the late-start LDRD was designed to screen a variety of ligand/metal complexes that a priori are believed to be more stable to polar solvents and possible mild hydrolytic conditions than are the phosphinoamido- ligands. 


\section{References}

1. $\quad$ Aresta, M.; Dibenedetto, A., Dalton Trans. 2007, 2975-2992.

2. $\quad$ Sakakura, T.; Choi, J.-C.; Yasuda, H., Chem. Rev. 2007, 107, 2365-2387.

3. Arakawa, H.; Aresta, M.; Armor, J. N.; Barteau, M. A.; Beckman, E. J.; Bell, A. T.; Bercaw, J. E.; Creutz, C.; Dinjus, E.; Dixon, D. A.; Domen, K.; DuBois, D. L.; Eckert, J.; Fujita, E.; Gibson, D. H.; Goddard, W. A.; Goodman, D. W.; Keller, J.; Kubas, G. J.; Kung, H. H.; Lyons, J. E.; Manzer, L. E.; Marks, T. J.; Morokuma, K.; Nicholas, K. M.; Periana, R.; Que, L.; Rostrup-Nielson, J.; Sachtler, W. M. H.; Schmidt, L. D.; Sen, A.; Somorjai, G. A.; Stair, P. C.; Stults, B. R.; Tumas, W., Chem. Rev. 2001, 101 (4), 953-996.

4. Song, C., Catal. Today 2006, 115, 2-32.

5. Jessop, P. G.; Joo, F.; Tai, C.-C., Coord. Chem. Rev. 2004, 248 (21-24), 24252442.

6. Miller, J. E.; Allendorf, M. D.; Diver, R. B.; Evans, L. R.; Siegel, N. P.; Stuecker, J. N., J. Mater. Sci. 2008, 43, 4714-4728.

7. Diver, R. B.; Miller, J. E.; Allendorf, M. D.; Siegel, N. P.; Hogan, R. E., J. Sol. Energy Eng. 2008, 130 (4), 041001.

8. http://en.wikipedia.org/wiki/Grignard_reaction.

9. Dell'Amico, D. B.; Calderazzo, F.; Labella, L.; Marchetti, F.; Pampaloni, G., Chem. Rev. 2003, 103, 3857-3897.

10. Tang, Y.; Kassel, W. S.; Zakharov, L. N.; Rheingold, A. L.; Kemp, R. A., Inorg. Chem. 2005, 44, 359-364.

11. Tang, Y.; Zakharov, L. N.; Rheingold, A. L.; Kemp, R. A., Organometallics 2004, 23 (20), 4788-4791.

12. Tang, Y.; Felix, A. M.; Manner, V. W.; Zakharov, L. N.; Rheingold, A. L.; Moasser, B.; Kemp, R. A., ACS Symp. Ser. 2006, 917 (Modern Aspects of Main Group Chemistry), 410-421.

13. Sita, L. R.; Babcock, J. R.; Xi, R., J. Am. Chem. Soc. 1996, 118 (44), 1091210913.

14. Kitamura, C.; Maeda, N.; Kamada, N.; Ouchi, M.; Yoneda, A., J. Chem. Soc., Perkin Trans. 1 2000, 781-785. 


\section{Distribution}

$\begin{array}{llllll}1 & \text { MS0359 } & \text { D. Chavez, LDRD Office } & \text { Org. } 01911 & \\ 1 & \text { MS0161 } & \text { Patent and Licensing Office } & \text { Org. } 11500 & \\ 1 & \text { MS0899 } & \text { Technical Library } & \text { Org. } 09536 & \text { (electronic) } \\ 8 & \text { MS1349 } & \text { R. A. Kemp } & \text { Org. } 01815 & \\ 3 & \text { MS1349 } & \text { J.E. Miller } & \text { Org. } 01815 & \\ 1 & \text { MS1349 } & \text { W. F. Hammetter } & \text { Org. } 01815 & \end{array}$

